\title{
Index of Equivalent Phonophorics
}

\begin{tabular}{|c|c|}
\hline $\begin{array}{l}\text { 毛, 鹿 ( 鹿) for *Maw } \\
\text { Paw }\end{array}$ & $\begin{array}{l}\text { [鹿], 表 (<Qin Seal 衰) biǎo *praw? ‘outer gar- } \\
\text { ment, express': 27, 28,\#009 }\end{array}$ \\
\hline 賁, 分 for ( ${ }^{\star}$ Pan $\left.<\right)^{\star}$ Par & [芬], 愤 fén *bər 'castrated pig': \#062 \\
\hline 夫, 甫 for *Pa & $\begin{array}{l}\text { [月夫], 述 bū *ppa ‘escape': 132, \#068 } \\
\text { [甫] as in 夫人 fū rén 'primary wife': } 131 \\
\text { Alternating in a word family for 'assist': 扶 fú } \\
\text { *ba 'support', 輔, 俌 fŭ *ba? 'assist' 'strut (of a } \\
\text { chariot)', 賻 fù *ba-s 'gift money', 傅 fù *pa-s } \\
\text { 'tutor': } 132\end{array}$ \\
\hline 父, 甫 for *Pa & $\begin{array}{l}\text { [須], [肸], [酺], 輔 fŭ *ba? 'cheeks, assist': 73, } \\
\text { 201, \#072 }\end{array}$ \\
\hline 畔 (半), 般 for *Pan & [繙], 盤 pán *bban 'waist band': 205, \#223 \\
\hline 伓, 北 for *Pək *Pə? & $\begin{array}{l}\text { [筫], 負 fù *bəP 'carry on the back' related to [伓], } \\
\text { 背 bèi *ppək-s 'the back': } 233, \# 078\end{array}$ \\
\hline 付, 部 for *Po & $\begin{array}{l}\text { [住], 附 fù *bo-s 'append, increase' and 蔀 bù } \\
\text { *bbo? 'thatch': 175, \#013 }\end{array}$ \\
\hline $\begin{array}{l}\text { 缶, 包 for *Pu *Paw } \\
\text { (dialect variation) }\end{array}$ & $\begin{array}{l}\text { [穏] piáo (according to Tangyun) *baw 'bag', 包 } \\
\text { bāo *ppru 'bag': } 223, \# 226\end{array}$ \\
\hline 龙, 蒙 for *Mon & $\begin{array}{l}\text { [龙], 蒙 méng*mmon related to máng*mmron } \\
\text { 'muddled', 'maculated': } 234, \# 199\end{array}$ \\
\hline 母, 某 for *Mə & $\begin{array}{l}\text { [畫], 謀 } m o ́ u \text { *mmə ‘plot’: } 180 \\
\text { [某], 梅 } m e ́ i \text { *mmə ‘plum’: } 180\end{array}$ \\
\hline 門, 昏 for *Mən & $\begin{array}{l}\text { [胝耳], [昏], 聞 wén, wèn *mən(-s) ‘hear, ask’: 27, } \\
\text { 209, 210, \#322 }\end{array}$ \\
\hline $\begin{array}{l}\text { 古 *Ka, 夫* }{ }^{*} \mathrm{~Pa}, \text { 甫 } * \mathrm{~Pa} \\
\text { (dialect variation) }\end{array}$ & 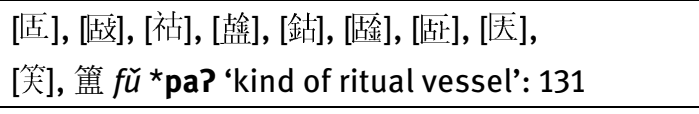 \\
\hline $\begin{array}{l}\text { 黃 *Kªn, 芷 *Kªn, } \\
\text { ᄃ *Pan (dialect variation) }\end{array}$ & 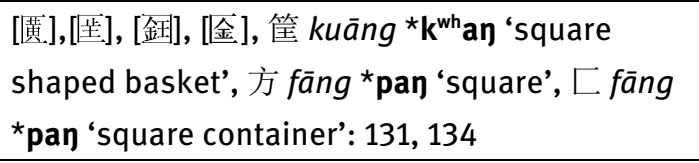 \\
\hline $\begin{array}{l}\text { 虎 }{ }^{\mathrm{Q}}(\mathrm{r}) \mathrm{a}, \text { 夫夫 }{ }^{\mathrm{Pa} a} \\
\text { (dialect variation) }\end{array}$ & [肤], 膚 $f \bar{u}$ *p(r)a ‘skin’: 224, \#068 \\
\hline
\end{tabular}




\begin{tabular}{|c|c|}
\hline $\begin{array}{l}\text { 享(亨) *Qan, 方 *Pan } \\
\text { (dialect variation) }\end{array}$ & [芳], 享 xiăng *q'an? ‘type of sacrifice’: 133 \\
\hline $\begin{array}{l}\text { 香 *Qan, 芳 *Pan } \\
\text { (dialect variation) }\end{array}$ & $\begin{array}{l}\text { 香 } x \bar{i} a n g \text { * } \mathbf{q}^{\text {h}} \text { an 'fragrance', 芳 fāng * } \mathbf{p}^{\text {h}} \text { an ‘fra- } \\
\text { grance': } 133\end{array}$ \\
\hline 卿 $\left(>\right.$ 饗), 享 $\sim$ 亨 for * ${ }^{\star}$ Qan & 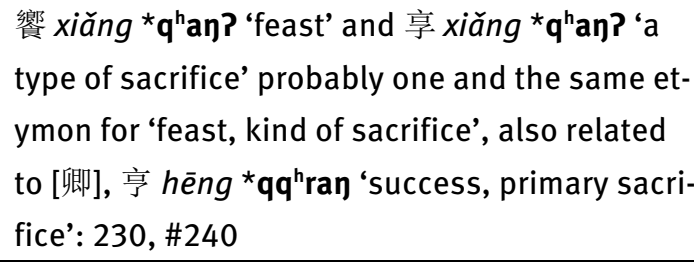 \\
\hline 舁, 車, 牙 for *K(r)a & 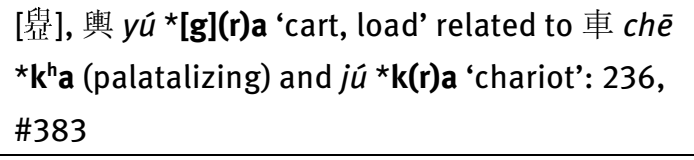 \\
\hline $\begin{array}{l}\text { 各, 假 for *Kak *Ka? } \\
\text { (dialect variation) }\end{array}$ & $\begin{array}{l}\text { [罟], [各], [垎], [迢] gé *kkrak 格 ‘arrive’ and [叞], } \\
\text { [徦], 假 jiǎ *kkra? ‘arrive’: 224, \#084 }\end{array}$ \\
\hline 閒/閒, 干 for *Kan * ${ }^{*}$ an & $\begin{array}{l}\text { [泊泊], 澗 jiàn *kkran-s 'valley’ related to 岸 àn } \\
\text { *nnan-s 'bank (of a river)': 232, \#146 }\end{array}$ \\
\hline 訐 (干), 蹇 for *Kan *Kat & $\begin{array}{l}\text { [訐] jié *kran 'reveal or bring up others' faults' } \\
\text { related to 赛 jiăn *kat 'pull up, lift': 237, \#152 }\end{array}$ \\
\hline 曷, 曳 for *Kat ${ }^{*}$ Qat & [遏], 曳 yè *[g]at 'drag': \#354 \\
\hline 㑆 , 衍 for *Kan & 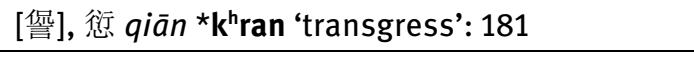 \\
\hline 堇, 艮, 斤 for $\left({ }^{*}\right.$ Kən $\left.<\right){ }^{*}$ Kər & $\begin{array}{l}\text { [墓], 艱 jiān *kkrər ‘be in difficult situation': } \\
\text { 238, \#143 related to [瞙], 限 xiàn *ggrər? (*N- } \\
\text { kkrər?) ‘obstacle, rough and dangerous (road)': } \\
238, \# 335 \\
\text { [斤], 謹 jǐn *kər? 'prudent’: } 83\end{array}$ \\
\hline $\begin{array}{l}\text { 堇, 斤 for *Tsam } \\
\text { (a second value) }\end{array}$ & $\begin{array}{l}\text { [澌], 漸 jian *dzam? ‘approach’: 82, \#147 } \\
\text { 漸 }\{\text { 水 }+(\text { 車 < }) \text { 堇 }+ \text { 斤 }\} \text { jiān *tsam ‘moisten’: } 82\end{array}$ \\
\hline 以, 已/已 for *Kə? & $\begin{array}{l}\text { [巳/已], 以 yǐ *[g]ə? ‘by means of’: 228, \#362, } \\
\# 363\end{array}$ \\
\hline 句, 拲 for *Ko & $\begin{array}{l}\text { [佝], 媾 gòu *kko-s ‘contract (a marriage)': 228, } \\
\# 093\end{array}$ \\
\hline 今, 欠 for *Kəm Qəm & $\begin{array}{l}\text { [酓], [㰸], 飲 yǐn *qəm? ‘drink’: 165, 205, \#368 } \\
\text { [舒], 琀, 含 hán ‘hold (in the mouth)’: 205, \#108 }\end{array}$ \\
\hline
\end{tabular}




\begin{tabular}{|c|c|}
\hline 高, 交 for *Kaw & $\begin{array}{l}\text { [蒿], [茭 ], [鄗], 郊 jiāo *kkraw 'suburb': 206, } \\
\text { \#149 }\end{array}$ \\
\hline 圭, 規 for ${ }^{*} K^{w} e$ & [閨], 闃 $k u \bar{i}{ }^{*} \mathbf{k}^{\mathrm{wh}} \mathbf{e}$ 'peep in’: \#173 \\
\hline 化, 骨 for * ${ }^{*}{ }^{w} a j ~ \sim ~ * Q^{w} a j$ & [货], 過 guò *kkwaj-s 'pass by’:\#106 \\
\hline $\begin{array}{l}\text { 花 }\left(\text { 化), 為 for * } K^{w} a j ~\right. \\
{ }^{\star} Q^{w} a j \sim \eta^{w} a j\end{array}$ & 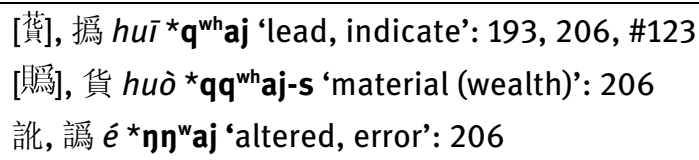 \\
\hline 戊, 月 for ${ }^{*} \mathrm{G}^{\mathrm{w}}$ at $\sim{ }^{*} \eta^{w}$ at & [或], 歲 sui *s-Gwat-s ‘year’: 144, \#297 \\
\hline 爱, 夗 for $\left({ }^{*} Q^{w} a n<\right){ }^{*} Q^{w} a r$ & $\begin{array}{l}\text { 媛 yuàn *Gwrar-s 'beautiful woman', 婉 wăn } \\
{ }^{*} \mathbf{q}^{\text {war? }} \text { 'beautiful': } 71\end{array}$ \\
\hline 官, 完 for *Kwan & 管, 筦 $g$ ǔan *kkwan? ‘pipe, flute’: 70 \\
\hline 元, 原 for $\left({ }^{*} \eta^{w} \text { an }<\right)^{*} \eta^{w}$ ar & 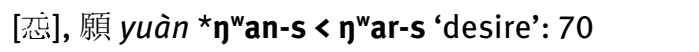 \\
\hline 堂, 威 for $Q^{w} \partial j$ & 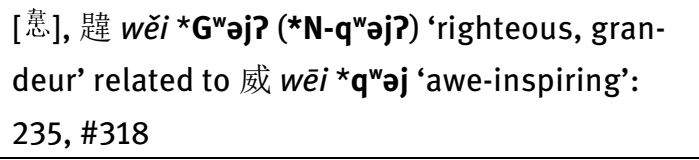 \\
\hline 吕/宮,弓 for *Kun & 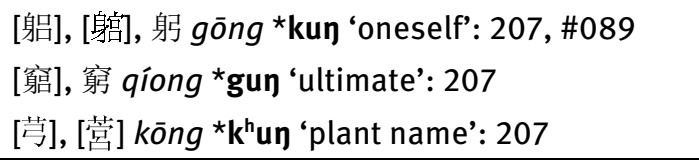 \\
\hline 率, 聿 for *S-rwət & $\begin{array}{l}\text { [街] (䢦), 帥 shuài *s-rwət-s 'lead' 'lead' related } \\
\text { to 率 ～律 lü *rwət 'rule, regulation':234, \#288 }\end{array}$ \\
\hline $\begin{array}{l}\text { 吝 }(\text { 文 }), \text { 粦 for }{ }^{\star} M-\text { rin } \sim * M- \\
\text { rən }\end{array}$ & [答], 鄰 lín *r[i/ə]n ‘neighbor’: 207, 208, \#186 \\
\hline 苗, 朝 for *M-raw & $\begin{array}{l}\text { [亩], 廟 miào *mraw-s ‘ancestral temple’: 27, } \\
\text { 183, \#203 }\end{array}$ \\
\hline $\begin{array}{l}\text { 隹, 离 for *Rwaj *Raj } \\
\text { (dialect variation) }\end{array}$ & $\begin{array}{l}\text { [羅] luó *rrwaj ‘bird trap’, ‘lay out’ and 離 lí *raj } \\
\text { 'bird name’ ‘lay out’: 225, \#194 }\end{array}$ \\
\hline 它, 彖 for (*Lwaj <)*Lwar & [㙫], 地 di *llwaj-s < *llwar-s 'ground': 173 \\
\hline 易, 狄 for * Lek & [易], 逷, 逖 ti *hllek ‘far’: \#365 \\
\hline $\begin{array}{l}\text { 帝 (=商), 易 for *Tek } \\
\text { *Lek (dialect variation) }\end{array}$ & $\begin{array}{l}\text { [甞], [帝], 惕 } t i{ }^{*} t^{\mathrm{h}} \text { ek *hllek 'alarmed': 189, } \\
\text { 220, \#300 }\end{array}$ \\
\hline 主, 斗 for *To? & [㪴], 斗 dǒu *tto? ‘Big Dipper’: 119, \#047 \\
\hline 東, 童, 重 for *Ton & [墥], [尰], 動 dòng *ddon? 'move’: 163 \\
\hline
\end{tabular}




\begin{tabular}{|c|c|}
\hline & $\begin{array}{l}\text { [僮], [墥], 童 tóng *ddon ‘adolescence': 163, } \\
\text { 164, \#306 } \\
\text { 鍾, 鐘 zhōng *ton ‘bell’: } 164\end{array}$ \\
\hline 竺 (竹), 畜 for *Tuk & [筀] \\
\hline 余, 予 for * La & $\begin{array}{l}\text { [俆], 序 xù *zl(r)a? ‘sequence’: 204, \# } 345 \\
\text { [余], 豫 yù *la-s ‘hexagram name’: 203, \#380 } \\
\text { 舒 shū *hla ‘comfortable’ written 豫, 念 in vari- } \\
\text { ous Shangshu versions: } 204\end{array}$ \\
\hline 尸, 夷 for * Lij & $\begin{array}{l}\text { [户], 夷 yí *lij ‘barbarian’: 212, 213, \#359 } \\
\text { 夷, [迡], 遲 chí *lrij ‘tardy’: 213, \#024 }\end{array}$ \\
\hline 卢, 敒 for * Lu & $\begin{array}{l}\text { [面], 迠], 做 yōu *lu 'object relativization } \\
\text { marker': } 209, \# 376\end{array}$ \\
\hline $\begin{array}{l}\text { 敒, 遙 for *Lu *Law } \\
\text { (dialect variation) }\end{array}$ & $\begin{array}{l}\text { 似, 悠 yōu *lu ‘far', 遙 yáo *law ‘far’: 222, \#415 } \\
\text { [榽], 繇, 由 yóu *lu ‘follow from': 223, \#352 }\end{array}$ \\
\hline $\begin{array}{l}\text { 由, 逐 for * }{ }^{*} \mathrm{u}(\mathrm{k}) \sim \text { * } \mathrm{Law}(\mathrm{k}) \\
\text { (dialect variation) }\end{array}$ & $\begin{array}{l}\text { 逐 zhú *lraw-k ‘pursue’, yóu *lu(k) 由 ‘follow } \\
\text { from’: 222, \#373 }\end{array}$ \\
\hline 同, 用/角 for *Lon & 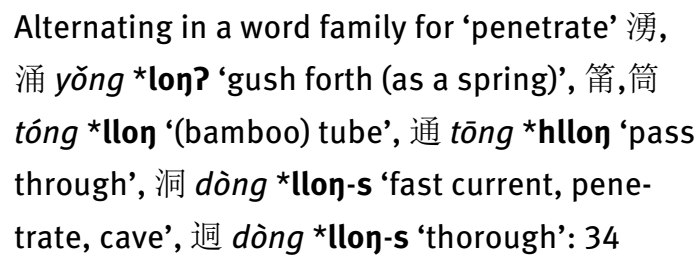 \\
\hline $\begin{array}{l}\text { 勺, 㷍 for * Lawk *Tawk } \\
\text { (dialect variation) }\end{array}$ & $\begin{array}{l}\text { [酌], 礿, 䄖 yuè, zhuó *lawk, *tawk ‘libation, } \\
\text { pour’: 226, \#393 }\end{array}$ \\
\hline $\begin{array}{l}\text { 翟, 侖 for *Lawk *Tawk } \\
\text { (dialect variation) }\end{array}$ & [鯩], 躍 yuè *lawk ‘jump’: 32, 227 \\
\hline 酨(才), 穴 for *Tsə & $\begin{array}{l}\text { [炎], [栽], [灾], 災 } z a \bar{a} i{ }^{*} \text { ttsə ‘natural disaster’: } \\
208, \# 395\end{array}$ \\
\hline 先, 次 for *Tsij & 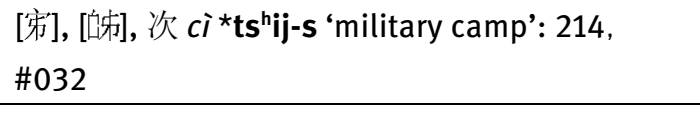 \\
\hline 妻, 齊 for *Tsij & 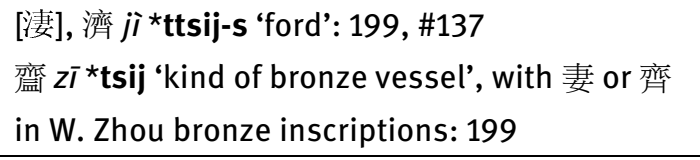 \\
\hline
\end{tabular}




\section{Two special classes of Equivalent Phonophorics}

EP for negative particles (from Section 5.2.3)

亡, 无 for wú * $\mathbf{m}(\mathbf{r}) \mathbf{a}$ 無 'not have, not exist': 215, \#311

母, 冊, 勿 for wù *mot 勿 'should not': 215, \#210, \#326

母, 冊, 未 for wèi *mot-s 未 'not yet': 216, \#210, \#319

勿, 無 for wú *m(r)a 無 'not have, not exist': 219, \#326

EP for sound-symbolic words (from Section 5.2.4)

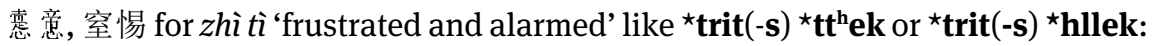
220, \#300, \#411

猷余, 由豫, 猷豫 for yóu yù 'being indecisive’ like ^lu ^la: 220, \#374, \#380

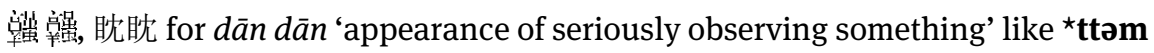
*ttəm or *ttwor *ttwor: 221, \#038

綪㱜, 鄞胥, 次且 for zī qiě ‘walk with difficulty’ like ${ }^{\star} \mathbf{t t s} \mathbf{~}_{\mathbf{i j j}}{ }^{\star} \mathbf{s r a}$ or ${ }^{\star} \mathbf{t s i j}{ }^{\star} \mathbf{t s}^{\mathrm{h}} \mathbf{a}$ ?: 221, \#287, \#418 
